\title{
Szenarios für x-Commerce
}

\author{
Gabriele Uchida \\ Institut für Statistik und Decision Support Systems \\ Universität Wien
}

Gerhart Bruckmann zum 70. Geburtstag gewidmet

\begin{abstract}
Zusammenfassung: Im Rahmen von ec3, dem Kompetenzzentrum für Electronic Commerce in Wien (Techgate) werden Anwendungsszenarien und Geschäftsmodelle für den Bereich x-Commerce untersucht, die den raschen Entwicklungen und den Veränderungen in diesem Bereich Rechnung tragen sollen.
\end{abstract}

\begin{abstract}
At ec3, a Competence-center for Electronic Commerce in Vienna (Techgate) applications scenarios and business models for $\mathrm{x}$-Commerce are studied to provide for rapid developments and changes in this areas.
\end{abstract}

Schlüsselwörter: Future Scenarios, Business Models, New Technologies and Services, Economic and Social Impact, Empirical Investigations on $\mathrm{x}$-Commerce.

\section{Einleitung}

Unter dem Begriff x-Commerce sollen Anwendungen aus den Bereichen e-Commerce (electronic commerce), m-Commerce (mobile commerce), c-Commerce (collaborative commerce), d-Commerce (demand-led commerce), k-Commerce (knowledge-based commerce), 1-Commerce (location based commerce), p-Commerce (pervasive commerce, public commerce, position commerce), s-Commerce (secure commerce), uCommerce (ubiquitous commerce) und eventuell andere vielleicht noch neu entstehende Commerce-Arten zusammengefasst werden. Besonderes Augenmerk sollte auf den Kontext mobiler Applikationen gelegt werden, insbesonders im Zusammenhang mit den Möglichkeiten, die sich durch die Verfügbarkeit aufkommender Breitbandtechnologien dabei abzeichnen.

Der Fokus soll dabei nicht auf den technologischen Aspekten liegen, sondern vor allem die sozialen, kulturellen, ökonomischen und rechtlichen Rahmenbedingungen und deren Auswirkungen berücksichtigen.

Der elektronische Geschäftsverkehr umfasst ein weites Spektrum verschiedener Tätigkeiten. Einige davon sind auch im klassischen Geschäftsverkehr wohl verankert, andere sind wiederum ganz neu (siehe European Commission, 1997). Auf Grundlage der neuen Technologien werden etliche Geschäftsprozesse dramatisch verändert, ermöglicht, beschleunigt, oder es entstehen sogar vollständig neuartige Formen. Diese teilweise dramatischen Änderungen sind nicht allein auf den privaten bzw. geschäftlichen Bereich beschränkt, sondern umfassen durchaus auch den öffentlichen Bereich (z.B. e-government). 
Es entstehen neue Wettbewerbs- und Kooperationsszenarien, ganz allgemein neue Rollendefinitionen, Verhaltensweisen und Interaktionen zwischen den beteiligten Parteien (z.B. Kunden, Organisation / Verwaltung, Kommunikations-Provider, Finanzdienstleister, Informationsdienstleister, Geschäftspartner, Händler, Vermittler etc.), sowohl innerhalb der Unternehmensprozesse als auch in Kommunikation und Austausch zwischen Anbietern, Mediatoren und Nachfragern. (siehe auch die 5 Wettbewerbskräfte bei Porter 1998).

In der Praxis werden jedoch neue, vielversprechende Technologien und Medien mit ihren enormen Potentialen oft ohne klares Konzept, ohne Studien zur User-Akzeptanz und die entsprechenden Auswirkungen eingesetzt und damit nicht unerhebliche Gefahren heraufbeschworen. Genaue Untersuchungen wie diese raschen Veränderungen der Geschäftsumgebung und der entsprechenden Strukturen Geschäftsprozesse beeinflussen scheinen nötig, um abschätzen zu können welche Geschäftsstrukturen in Zukunft nützlich und erfolgreich sein könnten.

Zum notwendigen Verständnis dieser neuartigen Strukturen und Gegebenheiten, werden sowohl empirische als auch theoretische Untersuchungen (Modellierungen) nötig werden. Einerseits wird man also aus vorliegendem oder noch $\mathrm{zu}$ erfassenden Datenmaterial Prognosen erstellen, meist allerdings ohne Berücksichtigung von Prozessabläufen, andererseits können Simulations-Modelle etwa in der Art von Unternehmensplanspielen, die ihren Fokus auf dem spielerischen Ertasten und Erlernen von Zusammenhängen in Form von Feedbackschleifen legen, zur Untersuchung neuartiger Szenarien herangezogen werden. Beide zusammen können auf ihre Art bei der Entscheidungsfindung helfen, Handlungsmöglichkeiten verdeutlichen und führen in weiterer Folge zur Entwicklung generischer Modelle.

\section{2 x-Commerce Szenarien}

\subsection{Rollen und Ziele}

Zunächst gilt es verschiedene Teilnehmer / Rollen im x-Business zu identifizieren und ihre entsprechenden Ziele und Verhaltensweisen zu verstehen. Im e-Business Kontext können sich die Akteure in verschiedenen Rollen wiederfinden. Ein Beispiel dazu wären etwa die sogenannten Umkehr-Auktionen, bei denen Einkäufer ihren Bedarf beschreiben und Lieferanten oder Dienstleister entsprechend anbieten können (z.B.: etwa Priceline, http://www.priceline.com/). Die Rollen sind nicht mehr so klar definiert wie im klassischen Business. Es wird oft schwierig Wettbewerb, Partner und Kunden zu unterscheiden. Ein Akteur kann ausserdem gleichzeitig oder kurz hintereinander Anbieter, Lieferant oder auch Vermittler (von Dienstleistungen, Gütern, Informationen) sein.

Mit den jeweiligen Rollen sind oft auch recht unterschiedliche Zielvorstellungen verbunden, von den klassischen Zielen wie etwa Gewinn-/Umsatzerhöhung, Kostenreduktion, Kundenbindung bis zu wenig greifbaren Zielen wie etwa Erhöhung des 
"Funfaktors". Speziell interessiert hier das Verhalten der User im Kontext von xCommerce Applikationen.

Folgende Fragen stellen sich dabei

- wie und warum werden neue Dienste akzeptiert bzw. nicht akzepiert?

- wieviel sind User bereit dafür zu bezahlen?

- wie kann man User halten?

- werden sich Neuentwicklungen rechnen?

- kann man aus vorhergehenden Erfolgen und Fehlschlägen für die Zukunft lernern?

\subsection{Nutzerverhalten}

Für jeden Anbieter von x-Commerce Applikationen ist das genaue Kennenlernen des Nutzerverhaltens von entscheidender Bedeutung. Die Akzeptanz einer Applikation kann etwa durch komplizierte Navigation, Brüche im Design, schwierige Abwicklung, teure oder unpraktische Endgeräte, unklare Kostenstrukturen sehr erschwert werden. Viele Nutzer sind nicht bereit, komplizierte Prozesse mitzumachen. Sie wechseln, sobald sie ein besseres oder vergleichbares Angebot finden. Meist geben sie auch kein direktes Feedback, sondern bleiben einfach weg und es ist dann oft nicht direkt nachvollziehbar was die Gründe für einen Misserfolg waren. Daher sollten x-commerce Applikationen gründlich getestet werden und natürlich immer aktuell sein. Usability tests (http://www.useit.com/) sollten selbstverständlich sein.

Wichtig erscheint es, Regeln für die Verhaltensweisen von - für x-commerce relevanten - Nutzergruppen zu finden, um Akzeptanzprognosen bei Einführung neuer oder bei Verbesserung bestehender x-commerce Anwendungen und Services erstellen zu können.

Dazu sind folgende Schritte nötig:

- Definition von x-commerce Nutzergruppen (Erstellung von Nutzerprofilen und -gruppen aufgrund von soziodemographischen Merkmalen, soziokulturellen Werten, Lebensstilen/Freizeitverhalten, aktuellen Lebensumständen)

- Definition von x-commerce Bereichen / Situationen (potentielle Anwendungsgebiete für $\mathrm{x}$-commerce Applikationen und Services werden durch die Bedürfnisse der Nutzer bestimmt)

- Definition von Verhaltensweisen / Regeln von Nutzergruppen in X-commerce Situationen (das Verhalten von Nutzergruppen wird in Relation zu bestimmten $\mathrm{x}$-commerce Situationen gesetzt)

Verschiedenste Methoden werden dazu verwendet:

- Nutzer-Befragungen: Online-Fragebögen, Panels, Fokusgruppen, ...

- Nutzer-Beobachtung: Logfile-Analyse, Webmining, Usertracking, Beobachtung in einem experimentellen Kontext (experimentelle Ökonomie), ... 


\subsection{Geschäftsmodelle}

Alt und Zimmermann (2001) weisen darauf hin, dass der Begriff "business model" oft erstaunlich undefiniert ist und versuchen durch Herausarbeiten von 6 "business model" Elementen etwas Struktur in den vorerst schwammigen Begriff zu bringen.

Timmers (1998) beschreibt den Begriff "business model” folgendermaßen:

"an architecture for the product, service and information flows, including a description of the various business actors and their roles; and a description of the potential benefits for the various business actors; and description of the sources of revenues."

In verschiedenen Arbeiten wird auch versucht, Geschäftsmodelle - besonders im elektronischen Kontext - zu klassifizieren und zu charakterisieren (siehe etwa Bartelt, Lamersdorf , 2001; Petrovic, Kittl, Teksten, 2001; Rappa, 2002; Timmers, 1998).

Durch die dynamischen Entwicklungen bleibt es eine grosse Herausforderung, Geschäftsprozesse in generischer Art und Weise zu beschreiben: Unternehmensgrenzen verschwimmen oft, Geschäftsprozesse verlassen die Unternehmensgrenzen. Partner, Lieferanten, andere Unternehmen müssen in bestehende Geschäftsprozesse integriert werden.

Ziel bleibt die elektronische Abwicklung von Geschäftsprozessen und die elektronische Informationsweitergabe über Organisations- bzw. Unternehmensgrenzen hinweg, um Kosten zu sparen und die Prozesse zu beschleunigen.

Die bisher bekannten Wertschöpfungsketten werden in vielen Fällen aufgebrochen und verkürzt (Picot und Heger, 2001). Beispiel: Der Verbraucher kauft direkt von Produzenten via Internet und der Zwischenhandel kann ausgeschaltet werden. In Zukunft sollte e-business durch grössere Flexibilität ein rascheres Erkennen und Reagieren auf Marktsignale und neu entstehende Geschäftsmodelle ermöglichen, sodass Anpassungen der Wertschöpfungskette schneller von statten gehen können.

Geschäftsmodelle im X-commerce Bereich haben oft deutlich andere Eigenheiten als herkömmliche Modelle:

- Information hat einen hohen Stellenwert

- Zugangs- und Nutzungsberechtigungen gewinnen gegenüber Handelsgütern an Bedeutung

- Produkte massgeschneidert

- Service personalisiert

- Communities gewinnen an Bedeutung

- Transaktionen ohne zeitliche Verzögerung durchführbar

Ex-post Untersuchungen bestehender Applikationen und deren Geschäftsmodelle und -prozesse sollen einen Überblick über Erfolgs- und Misserfolgsfaktoren geben, um daraus Parameter bzw. Faktoren für den erfolgreichen Einsatz neuer oder verbesserter xcommerce Applikationen und Services zu gewinnen und eventuell neuer Märkte und 
Zielgruppen zu identifizieren, wobei speziell die Bedürfnisse der zukünftigen Nutzer(gruppen) berücksichtigt werden sollen.

\section{Simulation}

Um all diese Aspekte untersuchen und abbilden zu können, soll ein Simulations-Modell (Szenario Modell) für x-commerce entwickelt werden. Angebots-Modelle, definiert durch bestehende oder geplante x-commerce Applikationen oder Services (Geschäftsmodelle, Geschäftsprozesse) werden mit Nachfrage-Modellen für potentielle Nutzer(gruppen) kombiniert.

Dazu sollen 3 Module entwickelt werden:

- Ein Expertensystem zur Nutzer-Analyse

- Das Simulations-System im engeren Sinn

- Ein Ergebnisanalyse-System

\subsection{Expertensystem Nutzer-Analyse}

Das Expertensystem Nutzer-Analyse soll aus einer Vielzahl von Datenquellen (Umfragen, Beobachtungen, Datamining, Studien, Online-Spiele) Nutzer-Spezifika extrahieren, etwa ihre Bedürfnisse, Werte, Verhalten, verschiedene Eigenschaften, aber auch Zielvorstellungen, sowohl strategisch als auch operativ. Im Anschluss daran werden daraus im Zusammenspiel mit Werte- und Prozessprofilen typische Nutzer für das Simulationssystem generiert.

\subsection{Simulations-System}

Abbildung 1 zeigt die Grobstruktur des Simulationssystems. Zur Simulation werden als Input sowohl Geschäftsmodell-Analysen, Branchen Information, besonders aber das Geschäftsprozessmodell einerseits und andererseits Nutzerdaten (Profil, Ziele, Verhalten) benötigt.

Ziel der Simulation soll sein, festzustellen inwieweit die verschiedenen Akteure in einer gegebenen Situation ihre Zielvorstellungen verwirklichen können, beziehungsweise welche Faktoren Zielerreichung be- oder verhindern.

\subsection{Ergebnisanalyse-System}

Die Ergebnisse der Simulation sollen nun kritisch gewürdigt und für den Nutzer des Systems verständlich aufbereitet werden. Hauptaugenmerk wird dabei darauf gelegt, welche Akteure in diesem System (also etwa Händler, Content Provider, Endkunde, ...) ihre Ziele in welchem Ausmaß verwirklichen konnten. Daraus wird dann eine Endbewertung des entsprechenden Szenarios vorgenommen, wobei eventuell auch Hindernisse oder Erfolgsfaktoren herausgearbeitet werden können. Abbildung 2 stellt die Struktur des Systems dar. 


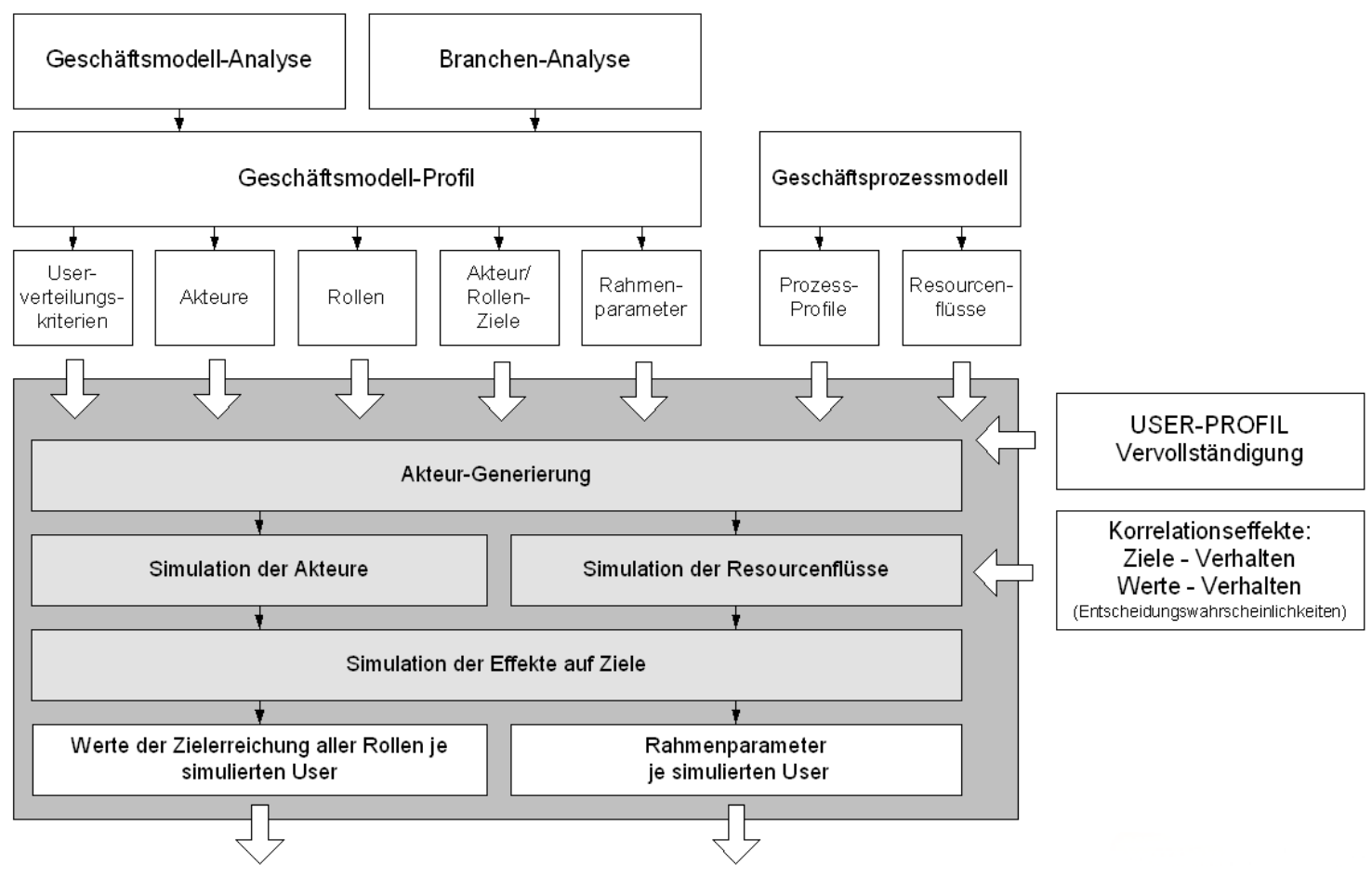

Abbildung 1: Struktur des Simulationsmodells

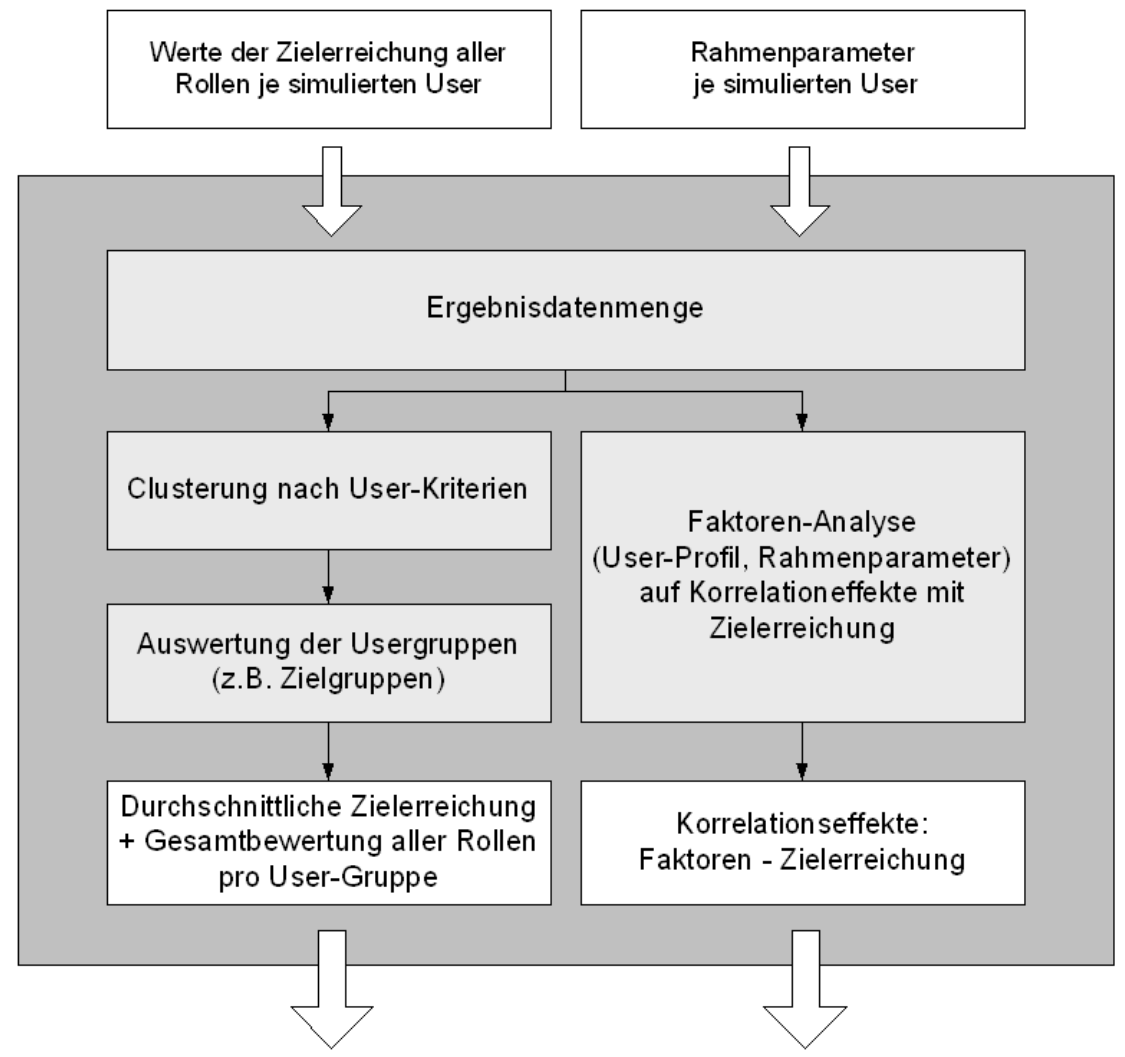

Abbildung 2: Struktur des Ergebnis-Analyse Systems 


\subsection{Zusammenfassung}

Wünschenswert wäre es, wenn solche Modelle etwa mögliche Einsatz-Barrieren oder Chancen für die Realisation neuer Applikationen vorhersagen könnte, um dadurch das Misserfolgs-Risiko von Neu-Einführungen zu verringern. Ähnlich den beliebten Unternehmensspielen, soll das „Ausprobieren“ verschiedener Szenarien und Parameterkonstellationen ein gefahrloses Abtesten der Auswirkungen wichtiger Entscheidungspfade ermöglichen und einen Überblick über die eventuell zu erwartenden Schwierigkeiten bieten.

\section{Danksagung}

Mein Dank gilt Markus Gratzer, Albert Hacker, Stefan Jager und Klaus Oberecker ohne deren Mithilfe diese Arbeit nicht zustande gekommen wäre.

\section{Literatur}

R. Alt and H.-D. Zimmermann. Preface: Introduction to special section - Business models, Electronic Markets, 11(1), 3-9, 2001. http://www.electronicmarkets.org/ netacademy/publications.nsf/all_pk/949.

A. Bartelt and W. Lamersdorf. A multi-criteria taxonomy of business models in electronic commerce, Proceedings of the IFIP/ACM International Conference on Distributed Systems Platforms (Middleware 2001), WS on Electronic Commerce, , pp. 193-205, Berlin Heidelberg, November 2001. Springer-Verlag.

European Commission. European Initiative in Electronic Commerce, $\mathrm{KOM}(97)$ 157, April 1997. http://www.cordis.lu/esprit/src/ecomcom.htm.

O. Petrovic, C. Kittl, and R.D. Teksten. Developing Business Models for eBusiness, Proceedings of the International Conference on Electronic Commerce, 31.10.20014.11.2001, Vienna, Austria.

A. Picot und D. Heger. Handel in der Internet-Ökonomie, Zeitschrift Führung und Organisation, 70(3):128ß134, 2001.

M. Porter. Competitive Advantage: Creating and Sustaining Superior Performance, New York, Free Press, 1998.

M. Rappa. Business Models on the Web, 2002. http://digitalenterprise.org/models/ models.html

P. Timmers. Business Models for Electronic Markets, Electronic Markets, 8(2):3-8, 1998. http://www.electronicmarkets.org/netacademy/publications.nsf/all_pk/184. 
Author's address:

Ao. Univ.-Prof. Dr. Gabriele Uchida

Institute for Statistics and Decision Support Systems

University of Vienna

Universitätsstraße 5

A-1010 Vienna, Austria

Tel. +4314277386/ 23

Fax +43 14277 - 9386

E-Mail: gabriele.uchida@univie.ac.at 